\title{
Critical Current Variation of Rutherford Cable of Bi-2212 in High Magnetic Fields with Transverse Stress*
}

\author{
D. R. Dietdericha , T. Hasegawa ${ }^{\text {, }}$ Y. Aoki ${ }^{\text {, }}$, and R. M. Scanlana \\ ${ }^{a}$ Lawrence Berkeley National Laboratory, \\ One Cyclotron Road, Berkeley, CA 94720, USA.

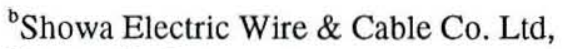 \\ Kawasaki, Japan.
}

For numerous high-energy physics applications superconducting cables are subjected to large stresses and high magnetic fields during service. It is essential to know how cables perform in these operating conditions. Transverse loading experiments on wire has shown that a significant drop in critical current occurs for stresses greater than $50 \mathrm{MPa}$. However, many applications require that the Bi-2212 conductor withstand stresses greater than $100 \mathrm{MPa}$ without permanent degradation. Therefore, a study of epoxy impregnated cables, identical to those used in accelerator magnet applications, has been performed. This work presents the first results of Rutherford cables of Bi-2212 with transverse stress. The results show that the cable can withstand stresses up to $60 \mathrm{MPa}$ with a strain of about $0.3 \%$ for the face loading orientation and $100 \mathrm{MPa}$ for the edge loading orientation.

\section{INTRODUCTION}

For the production of dipole fields above $15 \mathrm{~T}$, Bi-2212 may be an attractive alternative to $\mathrm{Nb}_{3} \mathrm{Sn}$. The main advantage is the low field dependence of $\mathrm{Bi}-2212$ in the $15-20 \mathrm{~T}$ range, where the $\mathrm{Nb}_{3} \mathrm{Sn} \mathrm{J}_{\mathrm{c}}$ is changing rapidly. However, before this advantage can be realized, the engineering Jc must be improved and the $\mathrm{J}_{\mathrm{c}}$ versus transverse strain behavior must be understood. This paper describes the first experiments aimed at understanding the $\mathrm{J}_{\mathrm{c}}$ versus transverse strain behavior of $\mathrm{Bi}-2212$ cables.

\section{EXPERIMENTAL DETAILS}

The testing procedure for the $\mathrm{Bi}-2212$ cables presented in this paper was the same as that reported previously for $\mathrm{Nb}_{3} \mathrm{Sn}$ cables. ${ }^{1}$ Briefly, the cables are insulated with S-glass, placed in the 304 stainless steel test tooling, and vacuum impregnated with CTD-101 epoxy. All of the measurements were made at the National High Magnetic Field Laboratory in Tallahassee, Florida, in a split-pair solenoid with a specially designed loading fixture in the solenoid bore which permits loads up to $500 \mathrm{kN}$ over a circular area $122 \mathrm{~mm}$ in diameter. This fixture and test geometry permits the magnetic field and the load to be applied normal to the current direction.

The cable used in this work, number 689, contained 20 strands of $\mathrm{Bi}-2212$ wire from billet number K288. It had a core of Ni-Cr 80 covered with two-layer wrap of $\mathrm{MgO}$ paper. The cable had the nominal dimensions of $2.240 \mathrm{~mm}$ thick and $8.890 \mathrm{~mm}$ wide. It was difficult to obtain a precise thickness measurement due to the soft $\mathrm{MgO}$ tape around the core. A thickness of $2.140 \mathrm{~mm}$ is obtained if the cable is firmly compressed during the measurement. Typically for $\mathrm{Nb}_{3} \mathrm{Sn}$ cable the compacted dimension is used when the cable is assembled into the reaction and test tooling. However, this was not the case for the Bi-2212 cables. During reaction the cable was not confined to its nominal dimensions in a reaction fixture since the cable expanded during reaction. Therefore, the post-reaction cable dimensions were used so that the conductor would not be strained during assembly in the test tooling. The post-reaction thickness and width were $2.348 \mathrm{~mm}$ and $8.94 \mathrm{~mm}$, respectively.

*This work was supported by the director, Office of Energy Research, Office of High Energy and Nuclear Physics, High Energy Physics Division, U.S. Department of Energy, under Contract No. DE-AC03-76SF00098. 
The cable was reacted on a large spool with a Diameter of $300 \mathrm{~mm}$. Insulation was placed between the layers to prevent sintering of adjacent cable. The cable was taken off of the reaction spool and placed on a spool for shipment from Showa to LBNL. The cable was unwound from the shipping spool, straightened, and placed into the test tooling. The calculated bending strain that the cable sustained was less than $1 \%$.

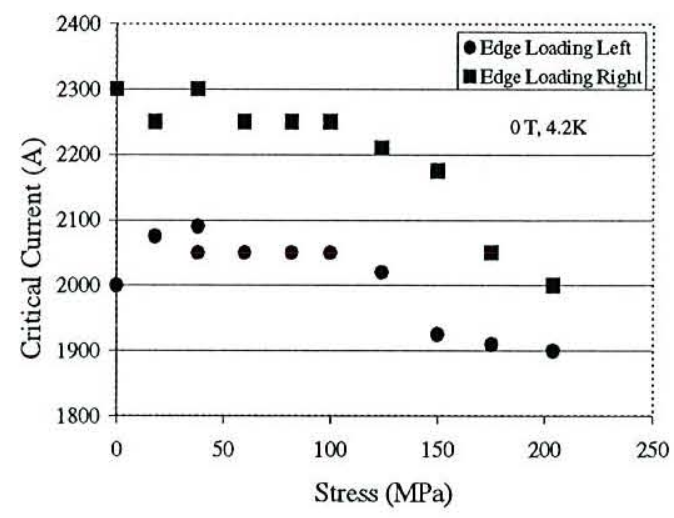

Figure 1. Variation of critical current (self-field, $4.2 \mathrm{~K}$ ) with stress for a cable that was edge loaded.

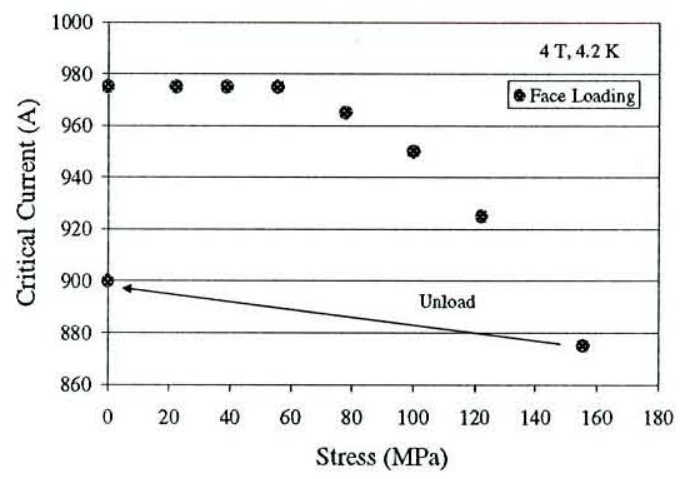

Figure 2. Variation of critical current (4 T, 4.2 K) with stress for a cable that was face loaded.

\section{RESULTS AND DISCUSSION}

The first measurements of the effect of transverse stress on the critical current of Bi-2212 wire has shown that the critical current dropped significantly for stresses as low as $10-20 \mathrm{MPa}^{2}$ This paper reports the first measurements of transverse stress on Rutherford cable. Figures 1 and 2 show that a significant drop in $\mathrm{I}_{\mathrm{c}}$ does not begin until the stress is greater than $60 \mathrm{MPa}$.
The $I_{c}$ of the cable measured in its self field (Fig. 1) is less than what one would expect from 20 times a single stand (i.e. 3,300 A). This decrease is more than one would calculate due to self-field effects in the cable, and the origin of the decrease is not known at this time. One source could be that the strands are damaged during cabling. However, previous results on strands that have been extracted from cable, heat-treated, and $I_{c}$ measured have shown no loss in current carrying capacity. ${ }^{3}$ The possibility of damage during assembly into the test tooling cannot be ruled out. This can only be determined by measurements on future cables. Another possible problem is the difficulty of processing a large sample of $\mathrm{Bi}-2212$ cable such that every section receives the optimum heat treatment.

An estimate of the overall strain of the face loading cable stack can be obtained. Mechanical modeling of the cable stack in the face loading orientation for a load of $100 \mathrm{MPa}$ gives a displacement of the steel beam into the cable package of about $25 \mu \mathrm{m}$. Using this value for the compaction of the stack and a stack thickness of 5 $\mathrm{mm}$ gives a strain of about $0.5 \%$. At $60 \mathrm{MPa}$ the strain would be about $0.3 \%$ which is about the irreversible strain limit for $\mathrm{Bi}-2223$ and $\mathrm{Bi}-2212$. $^{4,5}$ Comparison of these results and the work on wires show that a better method to extrapolate wire data to cables is required. In the future precise measurement of the strain in wire and cable that are being loaded transversely should facilitate the comparison.

\section{CONCLUSIONS}

These results show that $\mathrm{Bi}-2212$ conductor can withstand large stresses as long as the strand is well supported in a fiberglass-reinforced epoxy matrix. The important design parameter, as well as the best parameter for comparing strand and cable results, is the amount of local strain the strand undergoes.

\section{REFERENCES}

1. D. R. Dietderich, et al., IEEE Trans. Superconductivity, vol. 9 (1999) 122.

2. S. L. Bray, CEC-ICMC Montreal, 1999.

3. R. M. Scanlan, et al., IEEE Trans. Superconductivity, vol. 9 (1999) 130.

4. B. ten Hakken et al., Adv. in Cryo. Eng., 42A (1997), pp. 651-658.

5. B. ten Hakken et al., IEEE Trans on Magn., 32 (1996), pp. 2739-2742. 